\title{
Extracellular Vesicles Derived from Plasmodium-infected and Non-infected Red Blood Cells as Targeted Drug Delivery Vehicles
}

Livia Neves Borgheti-Cardoso a,b,", Sander A.A. Kooijmans ${ }^{c}$, Lucía Gutiérrez Chamorroa,b, Arnau Biosca $a, b^{a, b}$ Elena Lantero ${ }^{a, b}$, Miriam Ramírez ${ }^{b}$, Yunuen Avalos-Padilla ${ }^{a, b}$, Isabel Crespo ${ }^{d}$, Irene Fernández ${ }^{\mathrm{e}}$, Carmen Fernandez-Becerrab,f, Hernando A. del Portillob,f,g, Xavier Fernàndez-

$$
\text { Busquets }^{\mathrm{a}, \mathrm{b}, \mathrm{h},{ }^{*}}
$$

${ }^{a}$ Nanomalaria Group, Institute for Bioengineering of Catalonia (IBEC), The Barcelona Institute of Science and Technology (BIST), Barcelona, Spain

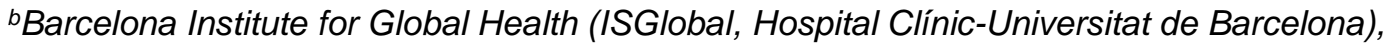
Barcelona, Spain

${ }^{c}$ Department of Clinical Chemistry and Haematology, University Medical Center Utrecht, Utrecht, The Netherlands

dPlataforma de Citometria, Institut d'Investigacions Biomèdiques August Pi i Sunyer (IDIBAPS), Barcelona, Spain

eUnitat d'Espectrometria de Masses de Caracterització Molecular ${ }_{\perp}$ CCiTUB, Universitat de Barcelona (UB), Barcelona, Spain

fInstitut d'Investigació en Ciències de la Salut Germans Trias i Pujol (IGTP), Badalona, Spain gInstitució Catalana de Recerca i Estudis Avançats, Barcelona, Spain ${ }^{h}$ Nanoscience and Nanotechnology Institute (IN2UB, UB), Barcelona, Spain

${ }^{*}$ Corresponding authors:

E-mail addresses: Iborgheti@ibecbarcelona.eu,xfernandez busquets@ub.edu Centre Esther Koplowitz, planta 1, ISGlobal, Rosselló 149-153, ES08036, Barcelona, Spain. 


\section{Abstract}

2 The mainAmong several factors behind drug resistance evolution in malaria isare the difficulty

3 challengeto of administering-appropriate overall doses that are not toxic for the patient but that,

4 locally, are sufficiently high to rapidly kill the parasites. Thus, a crucial antimalarial strategy is

5 the development of drug delivery systems capable of targeting antimalarial compounds to

6

Plasmodium with high specificity. as a result of the complex life cycle of Plasmodium, the short

7 half-lives of most antimalarial drugs, and the blood fluidic conditions affecting the interaction of

8 molecules with target cells. Extracellular vesicles (EVs) have been widely explored as delivery

9 vectors of nucleic acids and proteins. In the present study, extracellular vesicles (EVs) were

10 have been evaluated as a drug delivery system for the treatment of malaria. EVs derived from

11 naive red blood cells (RBCs) and from Plasmodium falciparum-infected RBCs ( $p R B C s$ ) were isolated by ultrafiltration followed by size exclusion chromatography. Lipidomic characterization showed that there weare no significant qualitative differences between the lipidomic profiles of pRBC-derived EVs (pRBC-EVs) and RBC-derived EVs (RBC-EVs). Both EVs were taken up by RBCs and pRBCs, although pRBC-EVs were more efficiently internalized than RBC-EVs, which suggested their potential use as drug delivery vehicles for these cells. When loaded into pRBCEVs, the antimalarial drugs atovaquone, lumefantrine and tafenoquine inhibited in vitro $P$. falciparum growth more efficiently than their free drug counterparts, indicating that pRBC-EVs can potentially increase the efficacy of several small hydrophobic drugs used for the treatment of malaria.

Keywords: Plasmodium falciparum; malaria; extracellular vesicles; drug delivery; antimalarial drugs.

\section{Introduction}

Malaria is a-serious disease of global health importance with nearly half of the world's population at risk of developing it. In 2018- occurred 228 million cases of malaria, which led to and an estimated 405,000 deaths, of which $67 \%(272,000)$ were children under 5 years of age (World $\underline{M}$ malaria Rreport 2019). One of the main difficulties in controlling malaria resides in the 
doses. There are several factors behind this problem, including (i) the formidable barrier imposed by the Plasmodium life cycle with its intracellular localization in hepatocytes and red blood cells (RBCs) (Balducci et al., 2016; Ofir-Birin et al., 2017), (ii) the physical environment of the circulatory system with strong flow drag and shear forces, affecting the interaction of drugs with target RBCs (Moles et al., 2015) and, (iii) the unfavorable physicochemical characteristics of antimalarial drugs, most of which are amphiphilic and widely distributed throughout the body tissues after administration. This leads to quick drug metabolism in the liver, resulting in short half-lives from a few hours to less than 1 hour (World Health Organization, 2015). In this scenario, the development of more efficient treatments for malaria is urgent. Frequently, the administration of antimalarial drugs occurs in a narrow therapeutic window between too high amounts inducing toxic side effects and too low local concentrations generating resistance (Balducci et al., 2016; Moles et al., 2015; World Health Organization, 2015). The administration of combinations of two or more drugs having different mechanisms of action and/or different biochemical targets in the parasite is the current recommended treatment to minimize resistance development (Balducci et al., 2016; Biosca et al., 2019; World Health Organization, 2015). However, rather than concentrating all efforts on discovering new drugs whose efficacy is quickly decreased by the parasite's capacity to develop resistance (Aditya et al., 2013; Fernàndez-Busquets, 2016), a crucial strategy is the development of targeted drug delivery systems capable of specifically delivering the antimalarial compound to Plasmodiuminfected RBCs (pRBCs), thus increasing the exposure of the parasite to doses sufficiently high to be lethal and minimizing the risk of drug resistance evolution (Fernàndez-Busquets, 2016; Moles et al., 2015).

Extracellular vesicles (EVs) have emerged as potential drug delivery systems to target specific organs or cells (Johnsen et al., 2014; Liao et al., 2019; Pinheiro et al., 2018; Stremersch et al., 2016; Tominaga et al., 2015; Vader et al., 2016). EVs have a size ranging from 40 to $1000 \mathrm{~nm}$ (El Andaloussi et al., 2013; Mantel and Marti, 2014; Raposo and Stoorvogel, 2013) and are released into the extracellular space by virtually all cells, thus being found in a large variety of body fluids including blood plasma, saliva, tears, urine, milk, and semen, among others (Liao et al., 2019; Raposo and Stoorvogel, 2013). EVs are heterogeneous in terms of size and cargo (Mardahl et al., 2019), containing cell-type-specific 
lipids, proteins, microRNAs and messenger RNAs that are essential for their function (Coakley et al., 2015; Mantel and Marti, 2014; Mardahl et al., 2019). Interestingly, many of these molecules are functional and can trigger phenotypic changes in recipient cells (Coakley et al., 2015; Mantel and Marti, 2014; Pinheiro et al., 2018). They are therefore believed to participate in intercellular communication and in both physiological and pathological processes (Raposo and Stoorvogel, 2013; Yáñez-Mó et al., 2015). Several studies have demonstrated that EVs play an essential role in parasite-host and parasite-parasite communication (Mantel and Marti, 2014; Marcilla et al., 2014; Mardahl et al., 2019; Ofir-Birin et al., 2017). During malaria infection, EV quantities in plasma were increased in patients when compared with healthy individuals, with the highest EV concentrations being observed in patients with severe Plasmodium falciparum malaria (Nantakomol et al., 2011; Pankoui Mfonkeu et al., 2010). Although these studies did not show a direct connection between higher EV levels and disease severity (Mantel and Marti, 2014), it was demonstrated in a mouse model that EVs derived from pRBCs induced systemic inflammation (Couper et al., 2010). In vitro studies also revealed that pRBCs release EVs which contain parasite and host cell proteins (Mantel et al., 2013; Regev-Rudzki et al., 2013), RNA and DNA (Mantel et al., 2016; Regev-Rudzki et al., 2013; Sisquella et al., 2017). These molecular cargos have potential roles in intercellular communication (Regev-Rudzki et al., 2013), modulation of immune response (Saxena et al., 2019; Sisquella et al., 2017), and parasite survival and malaria pathogenesis (Mantel and Marti, 2014; Mantel et al., 2013; Marcilla et al., 2014; Ofir-Birin et al., 2017; Regev-Rudzki et al., 2013). It was also shown that EVs derived from pRBCs can be internalized and transmit genetic material to other pRBCs (Mantel et al., 2013; Regev-Rudzki et al., 2013), monocytes (Sisquella et al., 2017) and endothelial cells (Saxena et al., 2019).

EVs present several advantages over synthetic delivery systems including (i) greater stability in the blood because of their natural surface composition (Kooijmans et al., 2012; Stremersch et al., 2016), (ii) possibly better protection of the encapsulated cargo due to a proteo-lipid architecture (Stremersch et al., 2016), (iii) endogenous cell and tissue targeting features afforded by their adhesion molecules and surface ligands (Kooijmans et al., 2012; Stremersch et al., 2016), (iv) higher biocompatibility allowing improved permeability through biological barriers, including the blood-brain barrier (Qu et al., 2018; Usman et al., 2018), and 
(v) almost nonimmunogenic character when used from autologous sources (Johnsen et al., 2014; Kao and Papoutsakis, 2019; Kooijmans et al., 2012; Liao et al., 2019; Lu et al., 2018; Pinheiro et al., 2018; Stremersch et al., 2016; Usman et al., 2018).

EVs from various-diverse cell types have been used as drug delivery systems for a variety of therapeutic agents (Vader et al., 2016), including both macromolecules (DNA, RNA (Alvarez-Erviti et al., 2011; Skog et al., 2008; Viñas et al., 2016) and proteins (Cho et al., 2018)) and small molecules (e.g. curcumin (Sun et al., 2010; Zhuang et al., 2011), doxorubicin (Lee et al., 2018; Tian et al., 2014), paclitaxel (Pascucci et al., 2014; Saari et al., 2015), methotrexate and cisplatin (Tang et al., 2012), photosensitizers (Silva et al., 2013), porphyrins (Fuhrmann et al., 2015) and dopamine (Qu et al., 2018)). Most of these studies focused on cancer therapy (Cho et al., 2018; Lee et al., 2018; Pascucci et al., 2014; Saari et al., 2015; Skog et al., 2008; Tang et al., 2012; Tian et al., 2014; Zhuang et al., 2011), but it was also shown that EVs can be used to treat other pathologies such as cerebral occlusion (Xin et al., 2013), Alzheimer's (Alvarez-Erviti et al., 2011) and Parkinson's disease (Qu et al., 2018), and ischemic kidney injury (Viñas et al., 2016). In the present study, EVs have been evaluated for the first time as drug delivery systems for a parasitic disease. EVs derived from pRBCs and non-infected RBCs were isolated and their size and composition were determined by bead-based flow cytometry assay, nanoparticle tracking analysis, cryogenic transmission electron microscopy, and lipidomic approaches. The targeting behavior of EVs towards RBCs and pRBCs was evaluated by flow cytometry. Finally, antimalarial drugs were loaded into EVs and their efficacy in inhibiting parasite growth was determined, showing that EVS have potential to treat parasitic diseases.

\section{Materials and Methods}

\subsection{Materials}

Unless otherwise indicated, all reagents were purchased from Sigma-Aldrich ${ }^{\circledR}$ (Saint Louis, USA).

\subsection{P. falciparum culture}

The $P$. falciparum 3D7 strain was grown in vitro in group B human erythrocytes as described previously (Cranmer et al., 1997). Briefly, parasites were cultured at $37^{\circ} \mathrm{C}$ in $\mathrm{T}-75$ flasks with 
RBCs at 3\% hematocrit in Roswell Park Memorial Institute (RPMI) complete medium containing $5 \mathrm{mg} / \mathrm{mL}$ Albumax II (RPMI-A, Life Technology, Auckland, New Zealand), supplemented with 2 mM L-glutamine (RPMI-AG), under a gas mixture of $92.5 \% \mathrm{~N}_{2}, 5.5 \% \mathrm{CO}_{2}$, and $2 \% \mathrm{O}_{2}$. Parasitemia was determined by microscopic counting of blood smears fixed briefly with methanol and stained with Giemsa (Merck Chemicals, Darmstadt, Germany) diluted 1:10 in Sorenson's buffer, $\mathrm{pH} 7.2$, for $10 \mathrm{~min}$. To obtain cultures synchronized in early ring stages $(0-24$ h post-invasion), a 5\% sorbitol lysis was performed (Lambros and Vanderberg, 1979). Late-form trophozoite and schizont stages (24-36 h and 36-48 h post-invasion, respectively) were selected in 70\% Percoll (GE Healthcare, Uppsala, Sweden) (Lambros and Vanderberg, 1979). For culture maintenance, parasitemia was kept at 3-5\% by dilution with freshly washed RBCs, and the medium was changed every 1-2 days.

\subsection{Isolation of EVs derived from RBCs and pRBCs}

EVs were isolated by centrifugation and ultrafiltration followed by size-exclusion chromatography (SEC). A previously described protocol (Díaz-Varela et al., 2018) was adapted for $\mathrm{EV}$ s isolation from either non-infected RBCs at 3\% hematocrit in RPMI-AG (48 $\mathrm{h}$ incubation) or $P$. falciparum cultured in RPMI-AG medium synchronized in ring stages and grown for $48 \mathrm{~h}$ at 2\%-of initial parasitemia (RBC-EVs and pRBC-EVs, respectively). Briefly, the culture was centrifuged at $400 \times \mathrm{g}$ for $10 \mathrm{~min}$ to remove cells, and the resulting EV-containing supernatant was centrifuged twice at $2,000 \times \mathrm{g}$ for $10 \mathrm{~min}$ to remove cell debris. To further concentrate EVs,

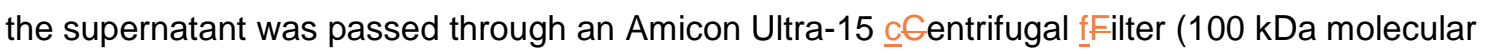
weight cut off; Millipore-Merck, Cork, Ireland). Finally, $1 \mathrm{~mL}$ of the resulting concentrate was loaded onto a Sepharose CL4B200 column (10 mL), which was pre-equilibrated with phosphate-buffered saline (PBS), and the purification was performed by gravity flow at room temperature (RT). 0.5-mL fractions were collected, and fractions 8 and 9 were used for the following experiments, after being concentrated using an Amicon Ultra-4 $\underline{\mathrm{c}}$ entrifugal fEilter

147 (100 kDa molecular weight cut off; Millipore-Merck). Two different Amicon Ultra çentrifugal fFilters with the same cut-off were used based on the initial volume of the samples and the desired volume of recovery. The protein concentration of EV suspensions in PBS was 
determined using a Pierce ${ }^{\mathrm{TM}}$ BCA Protein Assay Kit (ThermoScientific, Rockford, USA) according to the manufacturer's instructions.

\subsection{EV characterization}

\subsubsection{Bead-based flow cytometry}

Following a previously published protocol (de Menezes-Neto et al., 2015; Díaz-Varela et al., 2018), SEC fractions were analyzed by bead-based flow cytometry for the presence of the proteins glycophorin A (GPA) and histidine-rich protein 2 (HRP II), present in all RBCs and only in pRBCs, respectively. Briefly, SEC fractions were coupled to $4 \mu \mathrm{m}$-aldehyde/sulfate latex beads (Invitrogen, Carlsbad, USA) for $15 \mathrm{~min}$ at RT. Beads were then resuspended in $1 \mathrm{~mL}$ of bead-coupling buffer (BCB: PBS containing $0.1 \% \mathrm{BSA}$ and $0.01 \% \mathrm{NaN}_{3}$ ) and incubated overnight (RT, on rotation). EV-coated beads were then centrifuged $(2,000 \times \mathrm{g}, 10 \mathrm{~min}, \mathrm{RT})$ and the supernatant was removed, leaving the required sample volume to evaluate the different markers, before incubation with mouse monoclonal IgG anti-human GPA (Acris Antibodies, Herford, Germany) or mouse monoclonal anti-HRP II (Acris Antibodies) at 1:500 dilution for 30 min at $4{ }^{\circ} \mathrm{C}$. After washing with $\mathrm{BCB}, \mathrm{EV}$-coated beads were incubated for $30 \mathrm{~min}$ at $4{ }^{\circ} \mathrm{C}$ with Alexa Fluor $660^{\circledR}$-labeled goat anti-mouse secondary antibody (Invitrogen) at 1:500 dilution. Coated beads incubated with secondary antibodies only were used as a control. Labeled samples were analyzed by flow cytometry using a BD LSRFortessa flow cytometer (Becton, Dickinson and Company, New Jersey, USA), detected by excitation through a $640 \mathrm{~nm}$ laser and emission collection with a 710/50 nm bandpass filter; 20,000 beads per sample were examined and mean fluorescence intensity was determined using Flowing Software 2.5.1.

\subsubsection{Nanoparticle tracking analysis (NTA)}

The size and concentration of particles were determined at RT with a NanoSight LM10 system (Malvern Instruments Ltd) (de Menezes-Neto et al., 2015). Before analysis, EVs were diluted 2 to 100 times in PBS. For each measurement, three 60 -sec videos were recorded with the camera level set at 16. Data were analyzed with NTA software (version 3.1).

\subsubsection{Transmission electron microscopy (TEM)}


Knob-bearing erythrocytes infected with mature blood-stage $P$. falciparum parasites were purified from in vitro cultures in 70\% Percoll (Dluzewski et al., 1984) and fixed at $4{ }^{\circ} \mathrm{C}$ for $2 \mathrm{~h}$ with a mixture of $4 \%$ paraformaldehyde and $0.1 \%$ glutaraldehyde in $0.1 \mathrm{M}$ phosphate buffer, $\mathrm{pH}$ 7.4. Ultrathin cryosections were then obtained and processed as described elsewhere (Urbán et al., 2014), placed on Formvar ${ }^{\circledR}$-coated 200-hexagonal mesh nickel grids, and finally spiked, for focusing aid purposes, with an $\mathrm{lg} G$ coupled to $12 \mathrm{~nm}$ colloidal gold particles (Jackson ImmunoResearch). The observations were done in a Tecnai Spirit electron microscope. For cryogenic TEM (cryo-TEM), a thin film of pRBC-EVs and RBC-EVs was formed on a holey carbon grid and vitrified by plunging into ethane maintained at its melting point, using a Vitrobot (FEI Company, Eindhoven, The Netherlands). The vitreous films were transferred to a Tecnai F20 TEM (FEI Company), and the samples were observed in a low dose mode. Images were acquired with a CCD Eagle camera (FEI Company) at $200 \mathrm{kV}$ at a temperature of $-173^{\circ} \mathrm{C}$, using low-dose imaging conditions.

\subsubsection{Lipidomic analysis}

Lipid extraction was performed mixing $150 \mu \mathrm{L}$ of fraction 8 or 9 of SEC-purified RBC-EVs or pRBC-EVs in PBS with $150 \mu \mathrm{L}$ of chloroform:methanol (2:1), vigorously stirring for 2 min with a vortex. The resulting emulsion was centrifuged for $1 \mathrm{~min}$ at $16,000 \times \mathrm{g}$, and the lower phase containing the lipids was collected using a glass Pasteur pipette and stored at $4{ }^{\circ} \mathrm{C}$ until mass spectrometry analysis. Matrix-assisted laser desorption/ionization-time-of-flight (MALDI-TOF) mass spectra were recorded on a Sciex TOF/TOF 4800 MALDI mass spectrometer equipped with an Nd:YAG laser (355 nm). Time-of-flight mass analyses were acquired in reflectron and positive ion modes (low response was obtained in negative ion mode). One- $\mu \mathrm{L}$ aliquots of the extracted lipid solutions were mixed with $1 \mu \mathrm{L}$ of the matrix solution (2,5-dihydroxybenzoic acid saturated solution in acetonitrile), and $1 \mu \mathrm{L}$ of these mixtures was spotted on the sample plate and left to air dry before the analysis. Spectra were recorded from 500 to $1500 \mathrm{~m} / \mathrm{z}$ values and the dominant species were observed in the range of $650-850 \mathrm{~m} / \mathrm{z}$ values. Chemical identification of the most abundant lipids in the sample was done based on the $\mathrm{m} / \mathrm{z}$ values detected: $[\mathrm{M}+\mathrm{H}]^{+}$and $[\mathrm{M}+\mathrm{Na}]^{+}$for every compound, according to (Käkelä et al., 2003; Löhmann et al., 2010). The lipidic identification was confirmed based on major fragment ions of the 
MS/MS spectrum after performing a manual monoisotopic mass selection for every individual molecular species $\left(\mathrm{H}^{+}\right.$and $\mathrm{Na}^{+}$adducts $)$.

212

2.5. Cell targeting analysis

214

For pRBC targeting assays, pRBC-EVs and RBC-EVs were labeled with the amine-reactive fluorescent tag NHS-rhodamine (Thermo Scientific, Rockford, USA). Lyophilized dye was dissolved at a concentration of $10 \mathrm{mg} / \mathrm{mL}$ in dimethyl sulfoxide (DMSO), and $900 \mu \mathrm{L}$ of combined EV fraction 8 and 9 were mixed with $100 \mu \mathrm{L}$ of $100 \mathrm{mM}$ sodium bicarbonate $(\mathrm{pH}$ 8) containing $0.1 \mathrm{mg} / \mathrm{mL}$ NHS-rhodamine and incubated for $1 \mathrm{~h}$ at RT (Kooijmans et al., 2018). The unbound label was finally removed by SEC as described above. Fractions 8 and 9 were concentrated in Amicon Ultra-4 çCentrifugal fFilters (100 kDa cut off). _Labeled RBC-EVs (Rho-RBC-EVs) and pRBC-EVs (Rho-pRBC-EVs) were analyzed in a BD LSRFortessa flow cytometer. Forward- and side-scatter on a logarithmic scale and a mix of fluorescent beads of varied diameters (Megamix-Plus SSC, BioCytex, Marseille, France) were used to gate EVs. NHS-rhodamine fluorescence was detected by excitation through a $561 \mathrm{~nm}$ laser at $50 \mathrm{~mW}$ power and emission collection with a 582/15 nm bandpass filter. The acquisition was configured to stop after recording 20,000 events within the EV population. Rho-pRBC-EVs or Rho-RBC-EVs in PBS (protein content: $290 \mu \mathrm{g} / \mathrm{mL}$, ca. $9.7 \times 10^{8} \pm 0.2$ particles $/ \mathrm{mL}$ ) were added to desynchronized living $P$. falciparum 3D7 cultures and incubated for $24 \mathrm{~h}$. The cells were then stained for $10 \mathrm{~min}$ at RT with $4 \mu \mathrm{g} / \mathrm{mL}$ of the DNA dye Hoechst 33342, washed once (500×g, $5 \mathrm{~min}$ ) with RPMI-A, and resuspended in RPMI-AG. Samples were analyzed at $0.06 \%$ hematocrit in PBS in a BD LSRFortessa flow cytometer and also in an

232 imaging flow cytometer.

233 Forward- and side-scatter in a logarithmic scale were used to gate the RBC population. NHSrhodamine and Hoechst 33342 fluorescence were detected by excitation through 561 and 405 $\mathrm{nm}$ lasers at $50 \mathrm{~mW}$ power and emission collection with $582 / 15$ and $450 / 50 \mathrm{~nm}$ bandpass filters, respectively. The acquisition was configured to stop after recording 50,000 events within the RBC population. For imaging flow cytometry (Amnis ${ }^{\circledR}$ ImageStream ${ }^{\circledR X}$, Luminex). The acquisition 
debris, and out of focus events were excluded, gating the population of interest. Thereby, the population evaluated consisted of best focus single events.

\subsection{Growth inhibition assays}

pRBC-EVs that had been isolated by centrifugation and ultrafiltration followed by SEC from a 3day-old $P$. falciparum 3D7 culture $(0.5 \%$ initial parasitemia, late stages) were incubated in RPMI-AG for $24 \mathrm{~h}$ at RT without agitationstirring with two different concentrations of lumefantrine, tafenoquine, or atovaquone. A fresh P. falciparum 3D7 culture (6\% hematocrit and $1.2 \%$ parasitemia, ring stages) was seeded in $96-$ well plates and incubated for $48 \mathrm{~h}$ in the presence of the mixture of EVs (15.25 or $6.10 \mu \mathrm{g}$ protein $/ \mathrm{mL}$ ) and drugs (atovaquone at 0.085 and $0.17 \mathrm{nM}$, lumefantrine at 1.89 and $23.62 \mathrm{nM}$, and tafenoquine at 937.5 and $1875 \mathrm{nM}$ final concentrations). Parasite growth was determined in a BD FACSCalibur flow cytometer (Becton, Dickinson and Company, New Jersey, USA); samples were analyzed at $0.024 \%$ hematocrit in PBS, and the nuclei of pRBCs (the only nucleated cells present in the culture) were stained by addition of $0.1 \mu \mathrm{M}$ Syto11 (Invitrogen), added $10 \mathrm{~min}$ before analysis (Marques et al., 2016; Moles et al., 2015). Forward- and side-scatter on a logarithmic scale were used to gate the RBC population. Syto 11 green fluorescence was detected by excitation through aat $488 \mathrm{~nm}$-at (50 $\mathrm{mW}$ power) and emission collection with a $530 / 30 \mathrm{~nm}$ bandpass filter in the logarithmic scale. The acquisition was configured to stop after recording 50,000 events within the RBC population. Growth inhibition was determined by comparison of the parasite growth between treated and non-treated cultures.

A drug-free EV concentration range from 3 to $200 \mu \mathrm{g}$ protein $/ \mathrm{mL}$ was also evaluated for potential in vitro antimalarial activity. Cell-free RPMI-AG was processed identically as $\mathrm{PRBC}$ and RBC cultures for EV isolation, and the resulting EV-free fractions were used as control.

\subsection{Statistical analysis}

Differences between two data groups were analyzed using t-tests. Comparisons between more than two groups were made using one-way ANOVA with a Tukey post hoc test. Statistical differences were considered significant when $p<0.05$. 


\section{Results and Discussion:}

\subsection{EV isolation and characterization}

TEM images of $P$. falciparum 3D7 in vitro cultures revealed the presence of regions with abundant EVs in the close vicinity of pRBCs (Fig. 1). The concentration gradient observed, with a higher abundance of EVs near the plasma membrane and a thinning out of their numbers as the distance from the cell increased, was an indicator of the pRBC that was producing them.

To further characterize RBC- and pRBC-derived EVs, vesicles were isolated by combining ultrafiltration with SEC, a method which led to higher EV yield compared to ultracentrifugation (Nordin et al., 2015). SEC also offered a separation of free proteins in solution, increasing the purity of EVs (Vader et al., 2016). Moreover, the mild conditions of this method should better preserve the biophysical properties of EVs and allow them to retain functionality (Nordin et al., 2015; Stremersch et al., 2016; Vader et al., 2016)._Since EVs have surface proteins of the parental cell (Mardahl et al., 2019), EV-containing SEC fractions were identified by the presence of HRP II and of GPA. HRP II is a parasite protein expressed during most of the $P$. falciparum life cycle (Howard et al., 1986) and GPA is an abundant membrane protein present in all RBCs (Siebert and Fukuda, 1986) and both were previously identified in microvesicles derived from pRBCs (Mantel et al., 2013). According to bead-based flow cytometry assays, HRP II was best detected in SEC fractions 8 and 9 (Fig. 211A), whereas GPA showed a broader elution profile, and was most abundant in fractions 7, 8 and 9 for RBC-EVs, and in fractions $6,7,8$ and 9 for pRBC-EVs (Fig. 21B). Based on these results, fractions 8 and 9 were chosen for further experiments.

NTA revealed that RBC-EVs (fraction $8_{2} \div$ mean size $211.0 \pm 8.1 \mathrm{~nm}$; fraction $9_{2} \div$ mean size $202.2 \pm 5.6 \mathrm{~nm}$ ) were slightly larger than pRBC-EVs (fraction $8_{2}:$ mean size $179.7 \pm 6.8 \mathrm{~nm}$; fraction $9_{2} \div$ mean size $175.2 \pm 1.0 \mathrm{~nm}$ ) (Fig. 12C,-D, respectively). This was in contrast with the results reported by Mantel el at. (Mantel et al., 2013), where the majority of EVs derived from pRBCs and RBCs were sized between 100 and $150 \mathrm{~nm}$, a discrepancy that may be attributed to the use of a different EV isolation method (sucrose cushion ultracentrifugation). In $3 \%$ of hematocrit cultures incubated for $48 \mathrm{~h}, \mathrm{pRBC}$ produced more EVs than RBCs (Fig. 211C,-D), in agreement with data from previous studies (Mantel et al., 2013). The determined protein content was $103 \pm 41$ and $147 \pm 2 \mu \mathrm{g}$ protein/mL for RBC-EVs and pRBC-EVs, respectively. 

around $200 \mathrm{~nm}$ (Fig. 21E,-F), and the presence of mostly unilamellar smaller and larger EVs spanning a size range between ca. 100 and $400 \mathrm{~nm}$. Other structures not enclosed by a lipid bilayer, especially abundant in pRBC-EV preparations, were also revealed by cryo-TEM (Fig.

(303 21F).

\subsection{Lipidomic analysis of EVs}

306

Lipids are involved in the biogenesis and function of EVs, and some lipids are more abundant in EVs when compared to their parent cells. For example, sphingomyelins, cholesterol, and phosphatidylserine, which are the main components of lipid rafts, are especially abundant in EVs (Brzozowski et al., 2018).

_Proteomic analyses of RBC-EVs and pRBC-EVs are well described by Mantel et al. (Mantel et al., 2013). However, there is a lack of information regarding the lipid composition of pRBC-EVs and its potential alteration in pathological conditions (Brzozowski et al., 2018). Lipidomic analysis did not reveal significant qualitative differences between the lipid composition of EVs derived from RBCs and pRBCs (Fig. 32). For both EVs tThe most abundant lipids identified in both EVs were phosphatidylcholines (m/z 734.6, 756.6, 758.6, 760.6, 762.6, 780.6, 782.6, 784.6, 786.6, 788.6, 804.6, 806.6, 808.6, 810.6), and. S small amounts of sphingomyelins were also detected $(701.4,703.6,725.6,835.7,837.7)$. The nature of these compounds was confirmed in MS/MS analysis by the presence of the characteristic $\mathrm{m} / \mathrm{z}$ fragment of 184.1 Da derived from the phosphocholine headgroup (Löhmann et al., 2010). MS/MS ion fragmentation spectra of two phosphatidylcholines and two sphingomyelins are shown in the Supplementary Material|Information (Figs. S1 and S2, respectively). These results are consistent with the lipid composition of the RBC plasma membrane, where phosphatidylcholine and sphingomyelin are two of the main components (Quinn et al., 2009).

\subsection{Cellular uptake of EVs}

EV uptake is still a vexing question in the EV field because some studies demonstrated that the uptake of fluorescently labeled EVs can be done by practically every cell type evaluated (Svensson et al., 2013; Zech et al., 2012), whereas other reports proposed that EV take up only 
330 receptor (Mulcahy et al., 2014). Also, tit has been proposed that EVs can interact with target cells through several mechanisms such as direct fusion with the plasma membrane, endocytosis, binding to cell surface receptors and docking at the cell surface (Armstrong and Stevens, 2018; Kao and Papoutsakis, 2019; Mardahl et al., 2019; Pinheiro et al., 2018). Notably, the mechanism of EV interaction with cells, which depends in part on the recipient cell type (Kao and Papoutsakis, 2019), will influence their biodistribution and therapeutic potential (Pinheiro et al., 2018).

To determine the targeting behavior towards RBCs and pRBCs of EVs, these were labeled with NHS-rhodamine. EVs from RBC and pRBC were labeled with similar efficiency (70.1 $\pm 0.5 \%$ and $74.1 \pm 3.5 \%$, respectively).

\section{To investigate EV targeting to RBCs and $p R B C s, r$ Rhodamine-labeled EVs were} incubated for $24 \mathrm{~h}$ with an in vitro P. falciparum culture. Flow cytometry analysis revealed that both RBC- and pRBC-EVs bound RBCs and pRBCs (Fig. 4A3A,-B); pRBC-derived EVs were incorporated by both cell types with significantly higher avidity, and both vesicle types bound pRBCs significantly better than non-infected erythrocytes. In addition, EV targeting to RBCs and pRBCs using rhodamine-labeled EVs was also evaluated incubating pRBC-EVs for 15 min with an in vitro P. falciparum culture. EV uptake already occurred within this timeframe (Fig. S3).

The interactions of EVs with both RBCs and pRBCs could benefit their function as carriers of antimalarial drugs in drug delivery strategies. As shown in previous studies, delivering antimalarial drugs to non-infected RBCs could be an interesting strategy because Plasmodium would encounter a hostile environment immediately after invading the RBC, which would compromise its survival capacity (Moles et al., 2015). This approach resulted in a significantly improved efficacy of drugs encapsulated inside liposomes targeted to both pRBCs and RBCs (Moles et al., 2017, 2015). pRBC-EVs and RBC-EVs were incorporated by $100 \%$ of RBCs infected by Plasmodium late forms, whereas they were internalized by $87.1 \pm 1.4 \%$ and $76.9 \pm 1.9 \%$ of RBCs infected by Plasmodium ring forms, respectively (Fig. $4 \mathrm{AAA}$ ). The internalization of EVs by pRBCs containing early blood stages of Plasmodium is an attractive feature for the potential development of EVs as drug carriers, since delivering antimalarial compounds to parasite cells early in their intraerythrocytic cycle will contribute to reducing the viability of the pathogen (Moles et al., 2015). Using monocytes as targeting cells, Sisquella et al. 
demonstrated that pRBC-EVs showed a higher level of uptake by monocytes as compared to RBC-EVs (Sisquella et al., 2017). This suggests that target cells prefer uptake of EVs derived from infected cells over those from non-infected cells, possibly through the upregulation of specific surface markers on the pRBC-EVs which favourings interactions with the plasma membrane of target cells (Mardahl et al., 2019).

The internalization of pRBC-EVs by other pRBCs (Mantel et al., 2013; Regev-Rudzki et

al., 2013) and their role in intercellular communication affecting malaria pathogenesis have been described in the literature (Mantel et al., 2013; Regev-Rudzki et al., 2013; Saxena et al., 2019; Sisquella et al., 2017). However, to date, of our knowledge, there is no description of the role of EVs in physiological communication between RBCs. It has been claimed that EVs derived from group O RBCs can be used for large-scale EV production since they are readily available in blood banks and they are devoid of DNA (Usman et al., 2018), but EVs derived from

372 RBCs have been associated to activation of coagulation (Biró et al., 2003; Burger et al., 2013;

373 Rubin et al., 2013), endothelial activation (Straat et al., 2016b) and immunomodulation (Danesh 374 et al., 2014; Straat et al., 2016a), and thus could be involved in side effects that occur after 375 blood transfusion (Danesh et al., 2014; van Manen et al., 2019). In contrast with EVs produced

376 by circulating RBCs, EVs produced during RBC storage do not have detectable levels of 377 membrane markers associated with clearance, except for phosphatidylserine which was 378 exposed in low amount by a small fraction of EVs (van Manen et al., 2019). Imaging flow cytometry revealed an intracellular localization of EV-derived molecular components. Fig. $4 \mathrm{C}-\underline{3 \mathrm{C}}$ shows representative images of RBCs and pRBCs after uptake of rhodamine-labeled EVs, where a distribution of rhodamine-labeled EV molecular components throughout the cells can be observed. In parasitized erythrocytes, both RBC-EV- and pRBC-EVderived components colocalized with the nuclei of the parasites (Figs. $3 \mathrm{C}$ and $\mathrm{S} \underline{4} 3$ ).

Although this study shows the uptake of RBC-EVs and pRBC-EVs by RBCs and pRBCs, the mechanism by which this phenomenon occurs remains unclear. One could hypothesize that, since RBCs lack a typical endocytosis machinery, EV uptake occurs through fusion with the RBC membrane. Mechanistic studies of EV interaction with RBCs are still lacking, but assays done with liposomes, which share a phospholipid bilayer structure with EVs, may provide important clues. A proof of concept study evaluating liposomes containing quantum 
390

dots observed that liposomal contents entered the pRBCs through a process that likely involved fusion of the liposome lipid bilayer with the cell plasma membrane (Pujol et al., 2014; Urbán et al., 2011). In addition, phosphatidylcholine liposomes were shown to have better fusion/interaction with $\mathrm{pRBC}$ membranes compared with sphingomyelin liposomes (Hasan et al., 2011). As observed in the lipidomics study presented here, EVs derived from RBCs and pRBCs are rich in phosphatidylcholine, which could favor similar fusion processes, although it could also be speculated that structural and functional changes induced in RBCs by the parasite during its intraerythrocytic maturation (Hasan et al., 2011; Urbán et al., 2011) favor the higher uptake of EVs by pRBCs containing late forms of the parasite compared with pRBCs containing early blood stages of Plasmodium.

\subsection{Growth inhibition assays}

After evaluating the targeting behavior of EVs, which showed that pRBC-EVs had the most effective incorporation by RBCs and pRBCs, we next investigated the efficacy of pRBCEVs as a drug delivery system. Therapeutic agents can be loaded into EVs in different manners, which can be divided into two major strategies (Kao and Papoutsakis, 2019; Liao et al., 2019; Vader et al., 2016). In the first approach, usually employed to encapsulate RNA and proteins, these are loaded into the parent cells by transfection or incubation and later the cells release EVs containing the therapeutic agent. A second method consists of loading drugs into EVs after their purification, following one of several protocols involving co-incubation, electroporation, saponin treatment, sonication, extrusion and freeze-thaw cycles (Liao et al., 2019; Vader et al., 2016). Co-incubation is a simple and usually efficient method to load EVs with lipophilic drugs (Johnsen et al., 2014; Liao et al., 2019), which was used to encapsulate e.g. curcumin (Sun et al., 2010; Zhuang et al., 2011), paclitaxel (Saari et al., 2015) and dopamine (Qu et al., 2018). However, the therapeutic agents can leak out of the EVs in biological fluids, which can limit their applicability (Stremersch et al., 2016).

Here, we evaluated the loading of the antimalarial drugs lumefantrine, tafenoquine, and atovaquone in two different concentrations of pRBC-EVs (6.1으 or $15.25 \mu \mathrm{g}$ protein $/ \mathrm{mL})$ by simple incubation for $24 \mathrm{~h}$ at RT. These drugs were chosen due to their strong hydrophobic character (respective log $P$ values of 8.7, 5.0, and 5.2), which predicted their efficient 
incorporation in the lipid bilayer following incubation with EVs. We employed concentrations that, based on the IC50 of these compounds, would promote low inhibition of parasite growth

422 (Table 1). Non-encapsulated free drug was not removed from the samples because the different methods assayed to eliminate the drug not unincorporated drug inte EVs (ultracentrifugation, desalting columns, and SEC) were not able to clear the free compounds down to undetectable levels in in vitro $P$. falciparum growth inhibition assays, especially for the most potent antimalarials with lowest IC50 (data not shown). Therefore, we evaluated whether the fraction of drug encapsulated in PRBC-EVs could improve the therapeutic effect of the same amount of drug $100 \%$ in free form. Thus, the mixture of drug-loaded-EVs and the non-encapsulated free drug was added to synchronized P. falciparum 3D7 cultures, and growth inhibition was determined after $48 \mathrm{~h}$ by flow cytometry. As an additional control, pRBC-EVs without drug were included in the assay.

At their higher doses assayed, atovaquone and tafenoquine were more efficient in reducing the parasite viability in in vitro $P$. falciparum cultures when encapsulated in the higher pRBC-EVs concentration used (15.25 $\mu$ g protein $/ \mathrm{mL})\left(\right.$ Fig. $\left.\underline{4} 5 \mathrm{~A}_{2}=\mathrm{C}\right)$. This result suggests that more drug could be encapsulated when more EVs were available and indicates that pRBC-EVs intravesicular drug load. potentiate the effect of their drug cargo in inhibiting parasite growth, possibly through increased delivery to target cells. Saari et al. (Saari et al., 2015) also showed that EVs enhanced the cytotoxic effect of paclitaxel in a way directly proportional to the quantity of EVs and to the

At the lower drug doses tested, both tafenoquine and atovaquone encapsulated in the lower pRBC-EV concentration assayed $(6.1 \underline{0} \mu \mathrm{g}$ protein $/ \mathrm{mL})$ were more efficient than equal free drug amounts in decreasing parasite growth in an in vitro $P$. falciparum culture (Fig. 5A $\underline{4}-\mathrm{C}$ ). Nevertheless, a dose effect was not observed upon coincubation of lower drug doses with higher EV numbers, which suggests a likely saturation of pRBC-EV drug intake. At higher concentrations of EVs, however, these could still accommodate more drug in higher drug concentration assays. Lumefantrine offered the worst results at all drug and EV concentrations tested (Fig. 5B4B). It is conceivable that its high lipophilicity $(\log P=8.7)$ confers it a strong affinity for lipid bilayers, which might stabilize its permanence in the pRBC plasma membrane, where its antimalarial activity would be minor. 
It is also worth noting that tafenoquine is more potent inhibiting parasite growth at the

451 lower drug dose encapsulated into pRBC-EVs than at the higher drug dose (Fig. 56 4 C). Although there is no obvious explanation for this result, one can speculate a destabilizing effect on EV structure of tafenoquine when incorporated in the lipid bilayer of the vesicles. Such an effect has been recently described for the antimalarial drug domiphen bromide when inserted into liposomal membranes (Biosca et al., 2019). Tafenoquine has a relatively high molecular mass (ca. $582 \mathrm{Da}$ ), and it is likely that at high concentrations its bulk might destabilize the EV structure.

The same amounts of drug-free pRBC-EVs as those used in drug-containing samples had no effect on parasite growth (Fig. 5A4A-D). Drug-free RBC-EVs and pRBC-EVs started inducing significant inhibition of $P$. falciparum in vitro growth above $100 \mu \mathrm{g}$ protein $/ \mathrm{mL}$ (Fig. $5 \mathrm{D} \underline{\mathrm{D}})$. This result can be explained because large EV numbers will merge with the Plasmodium-infected RBC membrane and destabilize it, facilitating its rupture before the parasite cycle is complete; the resulting immature merozoites would likely have a decreased reinvasion capacity, which would result in a reduced parasite growth.

\section{Conclusion}

The current consensus is that a combination of different treatment strategies is required to eradicate malaria. In the drug therapy approach, it is important to have a drug delivery system that will efficiently encapsulate the drug and deliver it to the intended cell type. This will enable the use of overall reduced drug doses, with decreased adverse effects, but at the same time it will provide the capacity to deliver sufficiently high local amounts of drug to rapidly kill the parasite and thus limit drug resistance evolution. Here, we have isolated and characterized EVs from RBCs and pRBCs and explored them as antimalarial drug delivery vehicles. Both vesicle types showed potential as drug delivery carrier to RBCs and pRBCs because they were efficiently internalized by these cell types and the antimalarial drugstumefantrine, atovaquone and tafenoquine, when loaded in pRBC-EVs, more efficiently inhibited parasite growth than the free drug counterparts. Hence, pRBC-EVs could be an interesting candidate drug delivery system for certain lipophilic antimalarial drugs. 

gene transfer. In the case of RBCs, since they are devoid of both nuclear and mitochondrial DNA, it is claimed that EVs derived from these cells are potentially safer than EVs derived from nucleated cells (Usman et al., 2018). However, although RBCs lack DNA, they contain a small set of miRNA species that are present in EVs derived from them and which have been described to modulate target gene expression in endothelial cells (Mantel et al., 2016). Taken together, these reports counsel further investigation of the safety of pRBC-EVs and RBC-EVs as drug delivery vehicles.

487

\section{Acknowledgments}

This research was funded by the Ministerio de Ciencia, Innovación y Universidades, Spain (which included FEDER funds), grant numbers BIO2014-52872-R and RTI2018-094579-B-I00 (which included FEDER funds). LNB-C and YA-P were supported by the European Commission under Horizon 2020's Marie Skłodowska-Curie Actions COFUND scheme (712754) and by the Severo Ochoa programme of the Spanish Ministry of Science and Competitiveness (SEV-20140425 (2015-2019)). ISGlobal and IBEC are members of the CERCA Programme, Generalitat de

Catalunya. This research is part of ISGlobal's Program on the Molecular Mechanisms of Malaria which is partially supported by the Fundación Ramón Areces. The authors thank Joana

Marques for the preparation of $P$. falciparum cultures for TEM, and Inés Bouzón-Arnáiz for proteomic data analysis.

\section{Conflicts of interest}

The authors declare no competing financial interest.

\section{References}

Aditya, N.P., Vathsala, P.G., Vieira, V., Murthy, R.S.R., Souto, E.B., 2013. Advances in nanomedicines for malaria treatment. Adv. Colloid Interface Sci. 201-202, 1-17. https://doi.org/10.1016/j.cis.2013.10.014

Alvarez-Erviti, L., Seow, Y., Yin, H., Betts, C., Lakhal, S., Wood, M.J.A., 2011. Delivery of siRNA to the mouse brain by systemic injection of targeted exosomes. Nat. Biotechnol. 29, 341347. https://doi.org/10.1038/nbt.1807

Armstrong, J.P.K., Stevens, M.M., 2018. Strategic design of extracellular vesicle drug delivery systems. Adv. Drug Deliv. Rev. 130,12-16. https://doi.org/10.1016/j.addr.2018.06.017 
Balducci, A.G., Magosso, E., Colombo, G., Sonvico, F., 2016. From tablets to pharmaceutical nanotechnologies: Innovation in drug delivery strategies for the administration of antimalarial drugs. J. Drug Deliv. Sci. Technol. 32, 167-173. https://doi.org/10.1016/j.jddst.2015.06.003

Biosca, A., Dirscherl, L., Moles, E., Imperial, S., Fernàndez-Busquets, X., 2019. An ImmunoPEGliposome for targeted antimalarial combination therapy at the nanoscale. Pharmaceutics 11, 1-19.

Biró, É., Sturk-Maquelin, K.N., Vogel, G.M.T., Meuleman, D.G., Smit, M.J., Hack, C.E., Sturk, A., Nieuwland, R., 2003. Human cell-derived microparticles promote thrombus formation in vivo in a tissue factor-dependent manner. J. Thromb. Haemost. 1, 2561-2568. https://doi.org/10.1046/j.1538-7836.2003.00456.x

Brzozowski, J.S., Jankowski, H., Bond, D.R., McCague, S.B., Munro, B.R., Predebon, M.J., Scarlett, C.J., Skelding, K.A., Weidenhofer, J., 2018. Lipidomic profiling of extracellular vesicles derived from prostate and prostate cancer cell lines. Lipids Health Dis. 17, 1-12. https://doi.org/10.1186/s12944-018-0854-x

Burger, P., Kostova, E., Bloem, E., Hilarius-Stokman, P., Meijer, A.B., van den Berg, T.K., Verhoeven, A.J., de Korte, D., Van Bruggen, R., 2013. Potassium leakage primes stored erythrocytes for phosphatidylserine exposure and shedding of pro-coagulant vesicles. $\mathrm{Br}$. J. Haematol. 160, 377-386. https://doi.org/10.1111/bjh.12133

Cho, E., Nam, G., Hong, Y., Kyoung, Y., Kim, D.-H., Yang, Y., Kim, I.-S., 2018. Comparison of exosomes and ferritin protein nanocages for the delivery of membrane protein therapeutics. J. Control. Release 279, 326-335. https://doi.org/10.1016/j.jconrel.2018.04.037

Coakley, G., Maizels, R.M., Buck, A.H., 2015. Exosomes and other extracellular vesicles: The new communicators in parasite infections. Trends Parasitol. 31, 477-489. https://doi.org/10.1016/j.pt.2015.06.009

Couper, K.N., Barnes, T., Hafalla, J.C.R., Combes, V., Ryffel, B., Secher, T., Grau, G.E., Riley, E.M., De Souza, J.B., 2010. Parasite-derived plasma microparticles contribute significantly to malaria infection-induced inflammation through potent macrophage stimulation. PLoS Pathog. 6, e1000744. https://doi.org/10.1371/journal.ppat.1000744

Cranmer, S.L., Magowan, C., Liang, J., Coppel, R.L., Cooke, B.M., 1997. An alternative to serum for cultivation of Plasmodium falciparum in vitro. Trans. R. Soc. Trop. Med. Hyg. 91, 363-365.

Danesh, A., Inglis, H.C., Jackman, R.P., Wu, S., Deng, X., Muench, M.O., Heitman, J.W., Norris, P.J., 2014. Exosomes from red blood cell units bind to monocytes and induce proinflammatory cytokines, boosting T-cell responses in vitro. Blood 123, 687-696. https://doi.org/10.1182/blood-2013-10-530469

de Menezes-Neto, A., Sáez, M.J., Lozano-Ramos, I., Segui-Barber, J., Martin-Jaular, L., Ullate, J.M.E., Fernandez-Becerra, C., Borrás, F.E., del Portillo, H.A., 2015. Size-exclusion chromatography as a stand-alone methodology identifies novel markers in mass spectrometry analyses of plasma-derived vesicles from healthy individuals. J. Extracell. Vesicles 4, 27378. https://doi.org/10.3402/jev.v4.27378

Díaz-Varela, M., Menezes-Neto, A. de, Perez-Zsolt, D., Gámez-Valero, A., Seguí-Barber, J., Izquierdo-Useros, N., Martinez-Picado, J., Fernández-Becerra, C., Portillo, H.A. del, 2018. Proteomics study of human cord blood reticulocyte-derived exosomes. Sci. Rep. 8, 1-11. https://doi.org/10.1038/s41598-018-32386-2

El Andaloussi, S., Mäger, I., Breakefield, X.O., Wood, M.J.A., 2013. Extracellular vesicles: Biology and emerging therapeutic opportunities. Nat. Rev. Drug Discov. 12, 347-57. https://doi.org/10.1038/nrd3978

Fernàndez-Busquets, X., 2016. Novel strategies for Plasmodium-targeted drug delivery. Expert Opin. Drug Deliv. 13, 919-922. https://doi.org/10.1517/17425247.2016.1167038

Fuhrmann, G., Serio, A., Mazo, M., Nair, R., Stevens, M.M., 2015. Active loading into extracellular vesicles significantly improves the cellular uptake and photodynamic effect of 
porphyrins. J. Control. Release 205, 35-44. https://doi.org/10.1016/j.jconrel.2014.11.029

Hasan, G.M., Garg, N., Dogra, E., Surolia, R., Ghosh, P.C., 2011. Inhibition of the growth of Plasmodium falciparum in culture by stearylamine-phosphatidylcholine liposomes. J. Parasitol. Res. 2011, 1-9. https://doi.org/10.1155/2011/120462

Howard, R.J., Uni, S., Aikawa, M., Aley, S.B., Leech, J.H., Lew, A.M., Wellems, T.E., Rener, J., Taylor, D.W., 1986. Secretion of a malarial histidine-rich protein (Pf HRP II) from Plasmodium falciparum-infected erythrocytes. J. Cell Biol. 103, 1269-1277. https://doi.org/10.1083/jcb.103.4.1269

Johnsen, K.B., Gudbergsson, J.M., Skov, M.N., Pilgaard, L., Moos, T., Duroux, M., 2014. A comprehensive overview of exosomes as drug delivery vehicles - Endogenous nanocarriers for targeted cancer therapy. Biochim. Biophys. Acta 1846, 75-87. https://doi.org/10.1016/j.bbcan.2014.04.005

Käkelä, R., Somerharju, P., Tyynelä, J., 2003. Analysis of phospholipid molecular species in brains from patients with infantile and juvenile neuronal-ceroid lipofuscinosis using liquid chromatography-electrospray ionization mass spectrometry. J. Neurochem. 84, 10511065. https://doi.org/10.1046/j.1471-4159.2003.01602.x

Kao, C.-Y., Papoutsakis, E.T., 2019. Extracellular vesicles: Exosomes, microparticles, their parts, and their targets to enable their biomanufacturing and clinical applications. Curr. Opin. Biotechnol. 60, 89-98. https://doi.org/10.1016/j.copbio.2019.01.005

Kooijmans, S.A.A., Gitz-Francois, J.J.J.M., Schiffelers, R.M., Vader, P., 2018. Recombinant phosphatidylserine-binding nanobodies for targeting of extracellular vesicles to tumor cells: A plug-and-play approach. Nanoscale 10, 2413-2426. https://doi.org/10.1039/c7nr06966a

Kooijmans, S.A.A., Vader, P., van Dommelen, S.M., van Solinge, W.W., Schiffelers, R.M., 2012. Exosome mimetics: A novel class of drug delivery systems. Int. J. Nanomedicine 7, 15251541. https://doi.org/10.2147/IJN.S29661

Lambros, C., Vanderberg, J.P., 1979. Synchronization of Plasmodium falciparum erythrocytic stages in culture. J. Parasitol. 65, 418-420.

Lee, H., Park, H., Jin, G., Seong, E., 2018. pH-responsive hyaluronate-anchored extracellular vesicles to promote tumor-targeted drug delivery. Carbohydr. Polym. 202, 323-333. https://doi.org/10.1016/j.carbpol.2018.08.141

Liao, W., Du, Y., Zhang, C., Pan, F., Yao, Y., Zhang, T., Peng, Q., 2019. Exosomes: The next generation of endogenous nanomaterials for advanced drug delivery and therapy. Acta Biomater. 86, 1-14. https://doi.org/10.1016/j.actbio.2018.12.045

Löhmann, C., Schachmann, E., Dandekar, T., Villmann, C., Becker, C., 2010. Developmental profiling by mass spectrometry of phosphocholine containing phospholipids in the rat nervous system reveals temporo-spatial gradients. J. Neurochem. 114, 1119-1134. https://doi.org/10.1111/j.1471-4159.2010.06836.x

Lu, M., Xing, H., Xun, Z., Yang, T., Zhao, X., Cai, C., Wang, D., Ding, P., 2018. Functionalized extracellular vesicles as advanced therapeutic nanodelivery systems. Eur. J. Pharm. Sci. 121, 34-46. https://doi.org/10.1016/j.ejps.2018.05.001

Mantel, P.-Y., Marti, M., 2014. The role of extracellular vesicles in Plasmodium and other protozoan parasites. Cell. Microbiol. 16, 344-354. https://doi.org/10.1111/cmi.12259

Mantel, P.Y., Hjelmqvist, D., Walch, M., Kharoubi-Hess, S., Nilsson, S., Ravel, D., Ribeiro, M., Grüring, C., Ma, S., Padmanabhan, P., Trachtenberg, A., Ankarklev, J., Brancucci, N.M., Huttenhower, C., Duraisingh, M.T., Ghiran, I., Kuo, W.P., Filgueira, L., Martinelli, R., Marti, M., 2016. Infected erythrocyte-derived extracellular vesicles alter vascular function via regulatory Ago2-miRNA complexes in malaria. Nat. Commun. 7, 1-15. https://doi.org/10.1038/ncomms 12727

Mantel, P.Y., Hoang, A.N., Goldowitz, I., Potashnikova, D., Hamza, B., Vorobjev, I., Ghiran, I., Toner, M., Irimia, D., Ivanov, A.R., Barteneva, N., Marti, M., 2013. Malaria-infected erythrocyte-derived microvesicles mediate cellular communication within the parasite population and with the host immune system. Cell Host Microbe 13, 521-534. https://doi.org/10.1016/j.chom.2013.04.009 
Marcilla, A., Martin-Jaular, L., Trelis, M., de Menezes-Neto, A., Osuna, A., Bernal, D., Fernandez-Becerra, C., Almeida, I.C., del Portillo, H.A., 2014. Extracellular vesicles in parasitic diseases. J. Extracell. Vesicles 3, 25040. https://doi.org/10.3402/jev.v3.25040

Mardahl, M., Borup, A., Nejsum, P., 2019. A new level of complexity in parasite-host interaction: The role of extracellular vesicles, in: Advances in Parasitology. Elsevier Ltd., pp. 39-112. https://doi.org/10.1016/bs.apar.2019.02.003

Marques, J., Vilanova, E., Mourão, P.A.S., Fernàndez-Busquets, X., 2016. Marine organism sulfated polysaccharides exhibiting significant antimalarial activity and inhibition of red blood cell invasion by Plasmodium. Sci. Rep. 6, 24368. https://doi.org/10.1038/srep24368

Moles, E., Galiano, S., Gomes, A., Quiliano, M., Teixeira, C., Aldana, I., Gomes, P., FernàndezBusquets, X., 2017. ImmunoPEGliposomes for the targeted delivery of novel lipophilic drugs to red blood cells in a falciparum malaria murine model. Biomaterials 145, 178-191. https://doi.org/10.1016/j.biomaterials.2017.08.020

Moles, E., Urbán, P., Jiménez-Díaz, M.B., Viera-Morilla, S., Angulo-Barturen, I., Busquets, M.A., Fernàndez-Busquets, X., 2015. Immunoliposome-mediated drug delivery to Plasmodiuminfected and non-infected red blood cells as a dual therapeutic/prophylactic antimalarial strategy. J. Control. Release 210, 217-229.

Mulcahy, L.A., Pink, R.C., Carter, D.R.F., 2014. Routes and mechanisms of extracellular vesicle uptake. J. Extracell. Vesicles 3. https://doi.org/10.3402/jev.v3.24641

Nantakomol, D., Dondorp, A.M., Krudsood, S., Udomsangpetch, R., Pattanapanyasat, K., Combes, V., Grau, G.E., White, N.J., Viriyavejakul, P., Day, N.P.J., Chotivanich, K., 2011. Circulating red cell-derived microparticles in human malaria. J. Infect. Dis. 203, 700-706. https://doi.org/10.1093/infdis/jiq104

Nordin, J.Z., Lee, Y., Vader, P., Mäger, I., Johansson, H.J., Heusermann, W., Wiklander, O.P.B., Hällbrink, M., Seow, Y., Bultema, J.J., Gilthorpe, J., Davies, T., Fairchild, P.J., Gabrielsson, S., Meisner-Kober, N.C., Lehtiö, J., Smith, C.I.E., Wood, M.J.A., Andaloussi, S.E.L., 2015. Ultrafiltration with size-exclusion liquid chromatography for high yield isolation of extracellular vesicles preserving intact biophysical and functional properties. Nanomedicine 11, 879-883. https://doi.org/10.1016/j.nano.2015.01.003

Ofir-Birin, Y., Heidenreich, M., Regev-Rudzki, N., 2017. Pathogen-derived extracellular vesicles coordinate social behaviour and host manipulation. Semin. Cell Dev. Biol. 67, 83-90. https://doi.org/10.1016/j.semcdb.2017.03.004

Pankoui Mfonkeu, J.B., Gouado, I., Fotso Kuate, H., Zambou, O., Amvam Zollo, P.H., Grau, G.E.R., Combes, V., 2010. Elevated cell-specific microparticles are a biological marker for cerebral dysfunctions in human severe malaria. PLoS One 5, e13415. https://doi.org/10.1371/journal.pone.0013415

Pascucci, L., Coccè, V., Bonomi, A., Ami, D., Ceccarelli, P., Ciusani, E., Viganò, L., Locatelli, A., Sisto, F., Maria, S., Parati, E., Ester, M., Muraca, M., Alessandri, G., Bondiolotti, G., Pessina, A., 2014. Paclitaxel is incorporated by mesenchymal stromal cells and released in exosomes that inhibit in vitro tumor growth: A new approach for drug delivery. J. Control. Release 192, 262-270. https://doi.org/10.1016/j.jconrel.2014.07.042

Pinheiro, A., Silva, A.M., Teixeira, J.H., Gonçalves, R.M., Almeida, M.I., Barbosa, M.A., Santos, S.G., 2018. Extracellular vesicles: Intelligent delivery strategies for therapeutic applications. J. Control. Release 289, 56-69. https://doi.org/10.1016/j.jconrel.2018.09.019

Pujol, A., Urbán, P., Riera, C., Fisa, R., Molina, I., Salvador, F., Estelrich, J., FernàndezBusquets, X., 2014. Application of quantum dots to the study of liposome targeting in leishmaniasis and malaria. Int. J. Theor. Appl. Nanotechnol. 2, 1-8. https://doi.org/10.11159/ijtan.2014.001

Qu, M., Lin, Q., Huang, L., Fu, Yao, Wang, L., He, S., Fu, Yu, Yang, S., Zhang, Z., Zhang, L., Sun, X., 2018. Dopamine-loaded blood exosomes targeted to brain for better treatment of Parkinson's disease. J. Control. Release 287, 156-166. https://doi.org/10.1016/j.jconrel.2018.08.035

Quinn, P.J., Rainteau, D., Wolf, C., 2009. Lipidomics of the red cell in diagnosis of human 
disorders, in: Lipidomics, Methods in Molecular Biology. pp. 127-159. https://doi.org/10.1007/978-1-60761-322-0

Raposo, G., Stoorvogel, W., 2013. Extracellular vesicles : Exosomes, microvesicles, and friends. J. Cell Biol. 200, 373-383. https://doi.org/10.1083/jcb.201211138

Regev-Rudzki, N., Wilson, D.W., Carvalho, T.G., Sisquella, X., Coleman, B.M., Rug, M., Bursac, D., Angrisano, F., Gee, M., Hill, A.F., Baum, J., Cowman, A.F., 2013. Cell-cell communication between malaria-infected red blood cells via exosome-like vesicles. Cell 153, 1120-1133. https://doi.org/10.1016/j.cell.2013.04.029

Rubin, O., Delobel, J., Prudent, M., Lion, N., Kohl, K., Tucker, E.I., Tissot, J.D., AngelilloScherrer, A., 2013. Red blood cell-derived microparticles isolated from blood units initiate and propagate thrombin generation. Transfusion 53, 1744-1754. https://doi.org/10.1111/trf.12008

Saari, H., Lázaro-Ibáñez, E., Viitala, T., Vuorimaa-Laukkanen, E., Siljander, P., Yliperttula, M., 2015. Microvesicle- and exosome-mediated drug delivery enhances the cytotoxicity of Paclitaxel in autologous prostate cancer cells. J. Control. Release 220, 727-737. https://doi.org/10.1016/j.jconrel.2015.09.031

Saxena, R., Kaur, J., Hora, R., Singh, P., Singh, V., Mishra, P.C., 2019. CX3CL1 binding protein-2 (CBP2) of Plasmodium falciparum binds nucleic acids. Int. J. Biol. Macromol. 138, 996-1005. https://doi.org/10.1016/j.ijbiomac.2019.07.178

Siebert, P.D., Fukuda, M., 1986. Isolation and characterization of human glycophorin A cDNA clones by a synthetic oligonucleotide approach: Nucleotide sequence and mRNA structure. Proc. Natl. Acad. Sci. U. S. A. 83, 1665-1669. https://doi.org/10.1073/pnas.83.6.1665

Silva, A.K.A., Kolosnjaj-Tabi, J., Bonneau, S., Marangon, I., Boggetto, N., Aubertin, K., Clément, O., Bureau, M.F., Luciani, N., Gazeau, F., Wilhelm, C., 2013. Magnetic and photoresponsive theranosomes: Translating cell-released vesicles into smart nanovectors for cancer therapy. ACS Nano 7, 4954-4966. https://doi.org/10.1021/nn400269x

Sisquella, X., Yifat, O.-B., Pimentel, M.A., Cheng, L., Karam, P.A., Sampaio, N.G., Penington, J.S., Connolly, D., Giladi, T., Scicluna, B.J., Sharples, R.A., Waltmann, A., Avni, D., Schwartz, E., Schofield, L., Porat, Z., Hansen, D.S., Papenfuss, A.T., Eriksson, E.M., Gerlic, M., Hill, A.F., Bowie, A.G., Regev-Rudzki, N., 2017. Malaria parasite DNAharbouring vesicles activate cytosolic immune sensors. Nat. Commun. 8, 1985. https://doi.org/10.1038/s41467-017-02083-1

Skog, J., Würdinger, T., Rijn, S. Van, Meijer, D.H., Gainche, L., Sena-Esteves, M., Curry, W.T., Carter, B.S., Krichevsky, A.M., Breakefield, X.O., 2008. Glioblastoma microvesicles transport RNA and proteins that promote tumour growth and provide diagnostic biomarkers. Nat. Cell Biol. 10, 1470-1476. https://doi.org/10.1038/ncb1800

Straat, M., Böing, A.N., Tuip-De Boer, A., Nieuwland, R., Juffermans, N.P., 2016a. Extracellular vesicles from red blood cell products induce a strong pro-inflammatory host response, dependent on both numbers and storage duration. Transfus. Med. Hemotherapy 43, 302305. https://doi.org/10.1159/000442681

Straat, M., van Hezel, M.E., Böing, A., Tuip-De Boer, A., Weber, N., Nieuwland, R., van Bruggen, R., Juffermans, N.P., 2016b. Monocyte-mediated activation of endothelial cells occurs only after binding to extracellular vesicles from red blood cell products, a process mediated by $\beta$-integrin. Transfusion 56, 3012-3020. https://doi.org/10.1111/trf.13851

Stremersch, S., Smedt, S.C. de, Raemdonck, K., 2016. Therapeutic and diagnostic applications of extracellular vesicles. J. Control. Release 244, 167-183. https://doi.org/10.1016/j.jconrel.2016.07.054

Sun, D., Zhuang, X., Xiang, X., Liu, Y., Zhang, S., Liu, C., Barnes, S., Grizzle, W., Miller, D., Zhang, H.G., 2010. A novel nanoparticle drug delivery system: The anti-inflammatory activity of curcumin is enhanced when encapsulated in exosomes. Mol. Ther. 18, 16061614. https://doi.org/10.1038/mt.2010.105

Svensson, K.J., Christianson, H.C., Wittrup, A., Bourseau-Guilmain, E., Lindqvist, E., Svensson, 
L.M., Mörgelin, M., Belting, M., 2013. Exosome uptake depends on ERK1/2-heat shock protein 27 signaling and lipid raft-mediated endocytosis negatively regulated by caveolin-1. J. Biol. Chem. 288, 17713-17724. https://doi.org/10.1074/jbc.M112.445403

Tang, K., Zhang, Y., Zhang, H., Xu, P., Liu, J., Ma, J., Lv, M., Li, D., Katirai, F., Shen, G., Zhang, G., Feng, Z., Ye, D., Huang, B., 2012. Delivery of chemotherapeutic drugs in tumour cell-derived microparticles. Nat. Commun. 3, 1211-1282. https://doi.org/10.1038/ncomms2282

Tian, Y., Li, S., Song, J., Ji, T., Zhu, M., Anderson, G.J., Wei, J., Nie, G., 2014. A doxorubicin delivery platform using engineered natural membrane vesicle exosomes for targeted tumor therapy. Biomaterials 35, 2383-2390. https://doi.org/10.1016/j.biomaterials.2013.11.083

Tominaga, N., Yoshioka, Y., Ochiya, T., 2015. A novel platform for cancer therapy using extracellular vesicles. Adv. Drug Deliv. Rev. 95, 50-55.

Urbán, P., Estelrich, J., Cortés, A., Fernàndez-Busquets, X., 2011. A nanovector with complete discrimination for targeted delivery to Plasmodium falciparum-infected versus non-infected red blood cells in vitro. J. Control. Release 151, 202-211. https://doi.org/10.1016/j.jconrel.2011.01.001

Usman, W.M., Pham, T.C., Kwok, Y.Y., Vu, L.T., Ma, V., Peng, B., Chan, Y.S., Wei, L., Chin, S.M., Azad, A., He, A.B., Leung, A.Y.H., Yang, M., Shyh-Chang, N., Cho, W.C., Shi, J., Le, M.T.N., 2018. Efficient RNA drug delivery using red blood cell extracellular vesicles. Nat. Commun. 9, 1-15. https://doi.org/10.1038/s41467-018-04791-8

Vader, P., Mol, E.A., Pasterkamp, G., Schiffelers, R.M., 2016. Extracellular vesicles for drug delivery. Adv. Drug Deliv. Rev. 106, 148-156. https://doi.org/10.1016/j.addr.2016.02.006

van Manen, L., Peters, A.L., van der Sluijs, P.M., Nieuwland, R., van Bruggen, R., Juffermans, N.P., 2019. Clearance and phenotype of extracellular vesicles after red blood cell transfusion in a human endotoxemia model. Transfus. Apher. Sci. 58, 508-511. https://doi.org/10.1016/j.transci.2019.05.008

Viñas, J.L., Burger, D., Zimpelmann, J., Haneef, R., Knoll, W., Campbell, P., Vin, J.L., Gutsol, A., Carter, A., Allan, D.S., Burns, K.D., 2016. Transfer of microRNA-486-5p from human endothelial colony forming cell-derived exosomes reduces ischemic kidney injury. Kidney Int. 90, 1238-1250. https://doi.org/10.1016/j.kint.2016.07.015

World Health Organization, 2015. Guidelines For The Treatment of Malaria - 3rd edition. www.who.int/malaria 1-313. https://doi.org/10.1016/0035-9203(91)90261-V

World Malaria Report 2019, 202019. Geneva, World Health Organization. https://www.who.int/publications-detail/world-malaria-report-2019

Xin, H., Li, Y., Liu, Z., Wang, X., Shang, X., Cui, Y., Zhang, Z.G., Chopp, M., 2013. MiR-133b promotes neural plasticity and functional recovery after treatment of stroke with multipotent mesenchymal stromal cells in rats via transfer of exosome-enriched extracellular particles. Stem Cells 31, 2737-2746. https://doi.org/10.1517/17425247.2014.875528.Skin

Yáñez-Mó, M., Siljander, P.R.M., Andreu, Z., Zavec, A.B., Borràs, F.E., Buzas, E.I., Buzas, K., Casal, E., Cappello, F., Carvalho, J., Colás, E., Cordeiro-Da Silva, A., Fais, S., FalconPerez, J.M., Ghobrial, I.M., Giebel, B., Gimona, M., Graner, M., Gursel, I., Gursel, M., Heegaard, N.H.H., Hendrix, A., Kierulf, P., Kokubun, K., Kosanovic, M., Kralj-Iglic, V., Krämer-Albers, E.M., Laitinen, S., Lässer, C., Lener, T., Ligeti, E., Line, A., Lipps, G., Llorente, A., Lötvall, J., Manček-Keber, M., Marcilla, A., Mittelbrunn, M., Nazarenko, I., Nolte-'t Hoen, E.N.M., Nyman, T.A., O'Driscoll, L., Olivan, M., Oliveira, C., Pállinger, É., Del Portillo, H.A., Reventós, J., Rigau, M., Rohde, E., Sammar, M., Sánchez-Madrid, F., Santarém, N., Schallmoser, K., Ostenfeld, M.S., Stoorvogel, W., Stukelj, R., Van Der Grein, S.G., Helena Vasconcelos, M., Wauben, M.H.M., De Wever, O., 2015. Biological properties of extracellular vesicles and their physiological functions. J. Extracell. Vesicles 4, 1-60. https://doi.org/10.3402/jev.v4.27066

Zech, D., Rana, S., Büchler, M.W., Zöller, M., 2012. Tumor-exosomes and leukocyte activation: an ambivalent crosstalk. Cell Commun. Signal. 10,1-17. https://doi.org/10.1186/1478811X-10-37 
Zhuang, X., Xiang, X., Grizzle, W., Sun, D., Zhang, S., Axtell, R.C., Ju, S., Mu, J., Zhang, L., Steinman, L., Miller, D., Zhang, H.G., 2011. Treatment of brain inflammatory diseases by delivering exosome encapsulated anti-inflammatory drugs from the nasal region to the brain. Mol. Ther. 19, 1769-1779. https://doi.org/10.1038/mt.2011.164 